\title{
Drag model validation of slurry pipeline using CFD
}

\author{
Om Parkash*,**, Arvind Kumar** and Basant Singh Sikarwar*** \\ * Department of Mechanical Engineering, Amity University Haryana, Gurugram 122413, India \\ ** Department of Mechanical Engineering, J.C. Bose University of Science \& Technology, YMCA, Faridabad 121006, India \\ *** Department of Mechanical Engineering, Amity University Uttar Pradesh 201301, India \\ *,**Corresponding Author: om.mech8@gmail.com
}

Submitted : 22/10/2019

Revised : :19/01/2021

Accepted :29/01/2021

\begin{abstract}
A number of drag models have been suggested for the interaction of fluid particles in slurry flow over the previous centuries. It is necessary to examine the correctness and applicability of these models in the slurry transportation. Based on this concept, a comparative analysis of the different drag models is performed for the 0.0549 $\mathrm{m}$ diameter slurry pipeline. The research is carried out by using three drag models, Syamlal O'Brien, SchillerNaumann, and Gidaspow, due to their accessibility in the Fluent commercial software. The simulation is performed at mean flow velocity range, $V_{m}=2-5 \mathrm{~ms}^{-1}$ and solid concentration range, $C_{v \mathrm{f}}=10-20 \%$ (by volume) using computational drag models. The simulated outcomes for solid particle size $440 \mu \mathrm{m}$ having density $2470 \mathrm{~kg} / \mathrm{m}^{3}$ are recorded using Eulerian two-phase model with selected drag models in the computational domain. It has been found that the Eulerian two-phase model with Syamlal O'Brien drag model gives the accurate and meticulous results with the published data in the literature. Finally, the simulated outcomes of solid concentration contours, solid concentration profiles, and pressure drop are predicted at distinct velocity and solid concentration range for chosen drag models.
\end{abstract}

Keywords: 3D slurry pipeline; Drag models; Solid concentration; Pressure drop.

\section{INTRODUCTION}

Transportation of slurry flow through pipelines is a prevalent phenomenon that has been used for several centuries by different sectors around the globe. The hydraulic conveyance of solids in the form of slurry through pipelines includes primarily the sectors of food, pharmaceutical, chemical, oil, mining, cement and coal industries. This mode of solid transportation (i.e., coal ash, zinc tailing, copper tailing, sand, glass beads, iron ore slurry) has several advantages such as having no traffic, no pollution, and low maintenance, being environmentally friendly, and continuous delivery. Consequently, pipeline slurry conveyance is a secure and appealing mode of transport. However, the slurry flow through pipeline is a very complex phenomenon and is governed by a large number of variables in the pipeline. The factors affecting the slurry flow are particle size, flow rate, concentration of particles, etc. Therefore, the slurry flow behavior across the pipeline systems must be understood. Despite substantial studies using experimental information in this sector, slurry design engineers still face a lot of challenges. 
O'Brien (1933) and Rouse (1937) presented an open channel concentration distribution using a diffusion model for the low volume fraction of solids. Further, Ismail (1952) enhanced the diffusion model by relating the gradient of shear velocity to the coefficient of mass transfer. Ling et al. (2003) conducted numerical simulation of sand water blend flow in a horizontal straight pipe using algebraic slip model (ASM). The findings indicate motivating outcomes for mean slurry velocity and solid particulate settling velocity. Johansson et al. (2005) adopted closure and air feed models to simulate the fluidized bed through the pipeline. They predicted particle pressure drop and stress in the pipeline by using constant particle viscosity and granular flow theory, respectively. Their findings demonstrate that the kinetic theory of granular flow provides a more even distribution of bubble flow, while the constant particular viscosity provides the parabolic distribution of bubble flow. Kaushal et al. (2007) experimented near-wall lift force and pressure drop for coarser particulates and finer particulates respectively. They discovered that the effect of slip velocity on pressure drop was greater at higher velocity and less at lower velocity. Lahiri and Ghanta (2008) proposed a theoretical model by using support vector regression (SVR) and genetic algorithm (GA) to forecast the pressure drop in a solid-liquid mixture flow. Monteiro and Bansal (2010) discoursed the variation of pressure drop with ice concentration, pipe diameters, and slurry velocity using theoretical rheological model and predicted the performance of slurry flow parameters in the pipe. Majumder (2015) experimented glass beads and limestone mixture through inclined rectangular open channel and verified that the particle separation is a function of settling velocity. Assefa et al. (2017) proposed new empirical viscosity model for the highly concentrated Bingham slurry ranging from 50 to $70 \%$ by weight and found the proposed viscosity model in agreement with the experimental study for determining the viscosity of multisize particulates Bingham slurry flowing at higher concentration. B. B. Nayak and D. Chatterjee (2018) deployed mixture and Eulerian multiphase model strategies for predicting the transport characteristics of the fly-ash slurry qualitatively and quantitatively. Singh et al. (2018) simulated coal-water slurry through $90^{\circ}$ pipe bend and reported that pipe and bend radius ratio have great impact on head loss. Rabiei et al. (2018) proposed the simple methods for estimating the fluidic drag component during horizontal directional drilling operations. In their study, the installations validation was done with the FVM and three stage methods. In their study, they reported the new fluidic drag coefficient, and it was found that it changes linearly with the bore pipe length. Xu et al. (2018) simulated the ice particles in a pipe of shell and tube type heat exchanger of a polar ship. They investigated the effects of ice packing factor, ice particle diameter, and velocity on the distribution and melting characteristics of ice crystals. The findings of the paper reported the stratification of ice slurry flow and the degree of asymmetry for different ice particles and velocities.

Furthermore, Li et al. (2018) simulated the multisized particle mixture through a horizontal pipeline and predicted the slurry flow characteristics through it. The obtained outcomes revealed the changing trend of multisized particles through the pipeline at different solid concentration and flow velocity. Cai et al. (2019) predicted the ice slurry flow characteristics through the $90^{\circ}$ elbow using CFD-PBM coupled model. The findings show that the pressure drop increases with increase in flow velocity and ice packing factor. Liu at al. (2019) predicted the cemented paste backfill slurry transportation characteristics in consideration with hydration effects through the pipeline using mixture model and reported the three zones due to deposition of coarse and fine particles tailing in the pipeline. Ting et al. (2019) carried out the comparative analysis of coarser and fine particles by using CFD model and the Delft Head Loss \& Limit Deposit Velocity model for fully suspended slurry flow. Their study discovered the near wall effects of coarser and fine particles, and it was found that the CFD model accuracy decreases with the increase in particle size flow nearer to the wall. Singh et al. (2019) experimented the rheological behavior of bottom ash slurry and found that the sample containing 30\% and $40 \%$ concentration follows the Herschel-Bulkley model with nonNewtonian and shear thickening actions.

In the literature, numerous exploratory and numerical studies have been accounted for using different models for the parametric designing of slurry pipeline. However, it is found that the slurry pressure drop using different drag models is not available in the literature. The developed correlations and models available in the literature also led to 
great uncertainty because of their deviation from the experimental data. Additionally, the development of experimental setup and its validation with the drag model is quite tedious and time consuming. On the other hand, computational fluid dynamics possess ample scope and capability to evaluate the slurry flow characteristics for chosen drag models within a less time span. Therefore, a parametric study was conducted to analyze the slurry flow characteristics through the pipeline using the computational drag models, i.e., Syamlal O'Brien, Schiller-Naumann, and Gidaspow models. The velocity and solid concentration ranges of $2-5 \mathrm{~ms}^{-1}$ and $10-20 \%$ were used to obtain the simulated outcomes through the pipeline. Hence, the present study provides a reference to the slurry pipeline designer for the utility of different drag models in the transportation of slurry flow through pipeline system.

\section{MODEL DESCRIPTION}

\subsection{Eulerian Model}

Simulation is based on an effective Eulerian granular flow model comprising liquid and solid phases, i.e., $\alpha_{\mathrm{s}}+$ $\alpha_{1}=1$. The governing equations are solved for each phase, and coupling between the two phases is achieved through pressure and interphase exchange coefficients. The solid particle in the slurry flowis assumed to experience the following forces, i.e., static/solid pressure gradient $\left(\nabla P / \nabla P_{S}\right)$, viscous $\left(\nabla . \tau_{f}\right)$, and body forces $(\rho g)$, Lift/virtual forces, and forces due to phase velocities difference $\left(K_{s l}\left(v_{s}-v_{l}\right)\right)$. The lift and virtual mass coefficients $\left(\mathrm{C}_{\mathrm{L} /} C_{v m}\right)$ are assumed as 0.5 in the present study. The two phase Navier-Stokes equation has been settled to know the velocity and pressure field in the computational domain. The governing equations with appropriate assumptions are as follows:

\subsection{Governing Equatıons}

\section{Continuity Equation}

$\nabla \cdot\left(\alpha_{f} \rho_{f} v_{f}\right)=0$

Here, $f$ can be considered as $l$ for liquid phase and $s$ for solid phase

\section{Momentum Equations}

For liquid phase:

$$
\begin{aligned}
& \nabla \cdot\left(\alpha_{l} \rho_{l} v_{l} v_{l}\right)=-\alpha_{l} \nabla P+\nabla \cdot\left(\overline{\tau_{l}}+\overline{\overline{\tau_{f, l}}}\right)+\alpha_{l} \rho_{l} g+K_{s l}\left(v_{s}-v_{l}\right)+C_{v m} \alpha_{s} \rho_{l}\left(v_{s} \cdot \nabla v_{s}-v_{l} \cdot \nabla v_{l}\right) \\
& +C_{L} \alpha_{s} \rho_{l}\left(v_{l}-v_{s}\right) \times\left(\nabla \times v_{l}\right)
\end{aligned}
$$

Here, $K_{s l}$ represents the interphase momentum exchange coefficient, whereas $v_{l}$ and $v_{s}$ represent the velocity of liquid and solid phase, respectively.

For solid phase:

$$
\begin{aligned}
& \nabla \cdot\left(\alpha_{s} \rho_{s} v_{s} v_{s}\right)=-\alpha_{s} \nabla P-\nabla P_{s}+\nabla \cdot\left(\overline{\overline{\tau_{s}}}+\overline{\overline{\tau_{f, l}}}\right)+\alpha_{s} \rho_{s} g+K_{l s}\left(v_{l}-v_{s}\right)+C_{v m} \alpha_{s} \rho_{l}\left(v_{l} \cdot \nabla v_{l}-v_{s} \cdot \nabla v_{s}\right) \\
& +C_{L} \alpha_{s} \rho_{l}\left(v_{s}-v_{l}\right) \times\left(\nabla \times \mathrm{v}_{l}\right)
\end{aligned}
$$


Here, $\overline{\overline{\tau_{f, l}}}$ is the Reynolds stress tensor, whereas $\overline{\overline{\tau_{s}}}$ and $\overline{\overline{\tau_{l}}}$ are the viscous stress tensors for solid and liquid phase, respectively.

\subsection{Drag Models}

\section{Syamlal-O'Brien}

This model is applied for drag between particulate phases. This model is recommended for dense fluidized beds and thus depends on the particulate volume fraction. The interphase momentum exchange coefficient, $K_{s l}$ is given as

$$
K_{s l}=K_{l s}=\frac{3}{4} \frac{\alpha_{s} \alpha_{l} \rho_{l}}{V_{r, s}^{2} d_{p}} C_{D}\left(\frac{\operatorname{Re}_{s}}{V_{r, s}}\right)\left|v_{s}-v_{l}\right|
$$

Here, $\rho_{l}$ denotes the liquid phase density, and $d_{p}$ represents the diameter of solid particulate, which is assumed spherical in shape in the present study

The drag coefficient, $C_{D}$ is given as

$$
C_{D}=\left[0.63+4.8\left(\frac{\mathrm{Re}_{s}}{V_{r, s}}\right)^{\frac{-1}{2}}\right]^{2}
$$

The terminal velocity, $V_{r, s}$ for solid phase is given as

$$
\left.V_{r, s}=0.5\left(A-0.06 \operatorname{Re}_{s}+\sqrt{\left\{\left(0.06 \operatorname{Re}_{s}\right)^{2}+0.12 \operatorname{Re}_{s}(2 B-A)+A^{2}\right.}\right\}\right)
$$

where

$$
A=\alpha_{l}^{4.14} ; B=0.8 \alpha_{l}^{1.28} \text { for } \alpha_{l} \leq 0.85
$$

and

$$
A=\alpha_{l}^{4.14} ; B=\alpha_{l}^{2.65} \text { for } \alpha_{l}>0.85
$$

\section{Schiller-Naumann}

This model is used for modelling of drag between fluid phases in multiphase flow. The drag function ' $f_{d}$ ' is given as

$f_{d}=\frac{C_{D} \operatorname{Re}}{24}$ 
The drag coefficient $C_{D}$ is given as

$$
C_{D}= \begin{cases}24\left(1+0.15 \mathrm{Re}^{0.687}\right) / \mathrm{Re} & \mathrm{Re}<1000 \\ 0.44 & \mathrm{Re} \geq 1000\end{cases}
$$

The relative Reynolds number, $\mathrm{R}_{\mathrm{es}}$ between phases ' $l$ ' and ' $s$ ' is given as

$$
\operatorname{Re}_{s}=\frac{\rho_{l} d_{p}\left|v_{s}-v_{l}\right|}{\mu_{l}}
$$

Here, $\mu_{l}$ denotes the viscosity of the liquid phase

\section{Gidaspow}

This model is recommended for dense fluidized beds and thus depends on the particulate volume fraction.

$$
K_{l s}= \begin{cases}0.75 C_{D} \frac{\rho_{l} \alpha_{l} \alpha_{s}\left|v_{l}-v_{s}\right|}{d_{p}} \alpha_{l}^{-2.65} & \alpha_{l}>0.8 \\ \frac{150 \alpha_{s}\left(1-\alpha_{\mathrm{s}}\right) \mu_{l}}{\alpha_{l} d_{p}^{2}}+\frac{1.75 \rho_{l} \alpha_{l}\left|v_{l}-v_{s}\right|}{d_{p}} & \alpha_{l} \leq 0.8\end{cases}
$$

\section{COMPUTATIONAL DOMAIN AND GRID INDEPENDENT TEST}

In ANSYS 16.0, the computational mesh for a horizontal pipe of $3.8 \mathrm{~m}$ long and $0.0549 \mathrm{~m}$ diameter is generated. The pipe geometry comprises $462 \mathrm{k}$ hexahedral and quad elements, which concludes the suitable grid independent test. The grid independent test is performed at $C_{v f}=40 \%$ and $V_{m}=5 \mathrm{~ms}^{-1}$ for the pipe geometry containing $154 \mathrm{k}, 243$ $\mathrm{k}, 382 \mathrm{k}, 462 \mathrm{k}$, and $522 \mathrm{k}$ hexahedral and quad elements, where k represents the mesh elements in thousands. The solid phase velocity profile w.r.t pipe diameter in fully developed region for the distinctive mesh geometry is depicted in Fig.1 (b). It is found that the obtained velocity profile at aforementioned parameters overlaps for the pipe geometry containing $462 \mathrm{k}$ and $522 \mathrm{k}$ cells. Henceforth, a pipe geometry containing $462 \mathrm{k}$ cells is preferred for the simulation as portrayed in Fig. 1 (a). The minimum orthogonal quality and maximum ortho-skew representing the mesh quality are 0.93 and 0.065 , respectively. The computational pipe length is more than 50 diameters, which is adequately long for fully developed flow. 


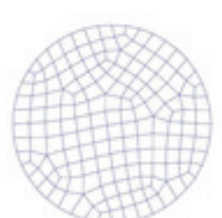

$154 \mathrm{k}$

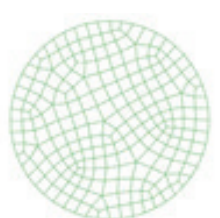

$243 \mathrm{k}$

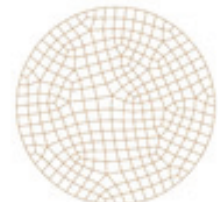

$382 \mathrm{k}$

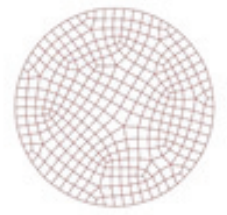

$462 \mathrm{k}$

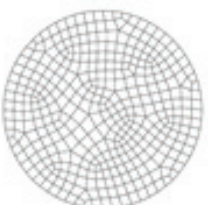

$522 \mathrm{k}$

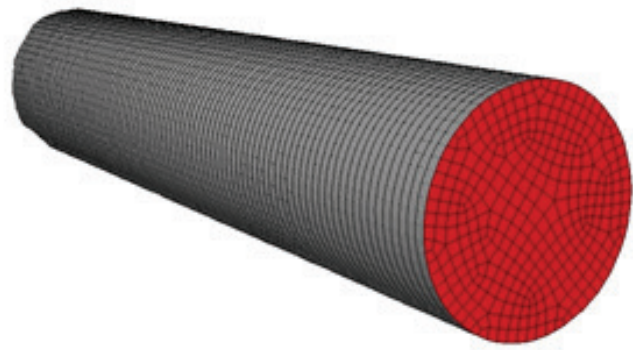

(a) Computational mesh

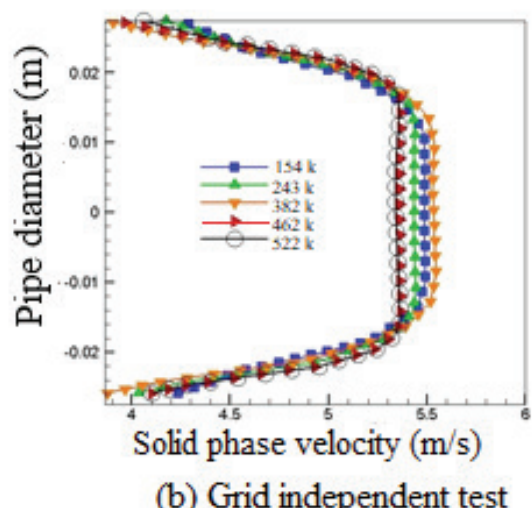

(b) Grid independent test

Figure 1. (a) Computational mesh and (b) Grid independence test (velocity profile with respect to radial distance at $V_{m}=5 \mathrm{~ms}^{-1}$ and $\left.C_{v f}=40 \%\right)$.

\subsection{Boundary Conditıons}

The pipeline geometry comprises three bounded faces viz. inlet, outlet, and wall boundaries to accomplish fluid domain calculations. The velocity inlet, pressure outlet, and no slip conditions are considered for the computational domain. The conditions for inlet/outlet boundaries are applied to the computational domain at particular velocity and volume fractions. The wall boundary is set to no slip conditions with a roughness constant 0.5 .

\subsection{Solution Methods and Convergence Criteria}

A $2^{\text {nd }}$ order upwind scheme is preferred in order to simplify the turbulence kinetic energy, volume fraction, momentum, and turbulence dissipation rate. The converging condition operates on the basis of residual value of various constraints viz. mass, turbulent kinetic, velocity, dissipation energy and volume fractions. The values for each constraint are pre-set to 0.001 times to the initial residual value. The solution control relaxation factor used are given as pressure -0.3 , momentum -0.5 , turbulent viscosity -0.8 , volume fraction -0.5 , turbulent kinetic energy and dissipation energy as 0.8 . This course of action offers high exactness, fidelity and convergence. 


\section{COMPUTATIONAL RESULTS}

\subsection{Solıd Concentration Contours}

Figures 2 depicts the solid concentration contours in the computational domain for mean flow velocity, $V_{m}=2$ $\mathrm{ms}^{-1}$ at solid concentration range, $C_{v f}=10-20 \%$. It is found that the solid particulates concentration is extreme in the lower, half section of the pipeline due to gravitational effect. However, the solid particulate concentration is varying from bottom to top of the pipeline for the chosen models. The reason for variation of solid concentration depends on particulate diameter, volume fraction, and near wall lift force for chosen models as depicted in figure 2 (a). Gidaspow and Syamlal O'Brien drag models are more suitable for fluidized bed flow in comparison to SchillerNaumann model, because Schiller-Naumann model is suitable for dilute phase. Moreover, the coupling of two phases in Gidaspow and Syamlal O'Brien drag models is gained through momentum exchange between the phases. Furthermore, as the solid concentration increases the thickness of the fluidized bed moving in the pipe bottom increases as depicted in figure 2 (b). In addition, as the velocity increases, the turbulence mixing increases, which enhances the momentum exchange between the solid particulates and the pipe wall that causes diminution in the thickness of the fluidized bed as depicted in figures 3 (a) and 3(b) at $C_{v f}=10$ and 20\%, respectively. The same effect can also be seen for velocity 3 and $4 \mathrm{~ms}^{-1}$ at $C_{v f}=10$ and $20 \%$ for the chosen drag models.

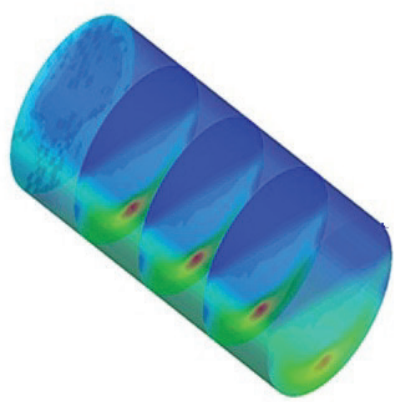

Syamlal - Obrien

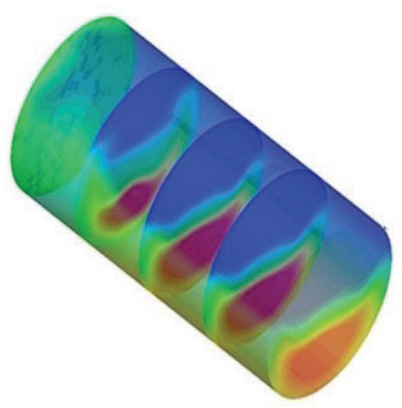

Syamlal - Obrien

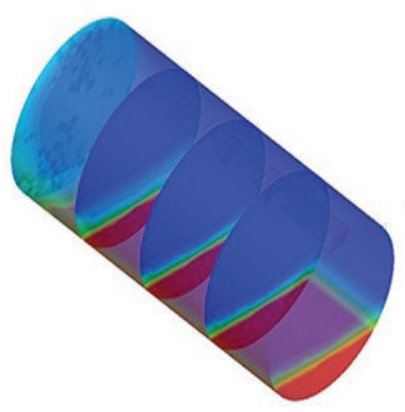

Schillar - naumann

(a) at $\mathrm{Cvf}=10 \%$

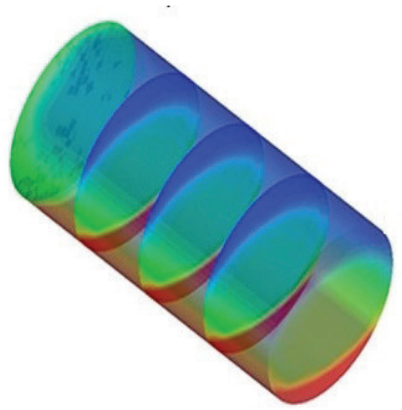

Schillar - naumann

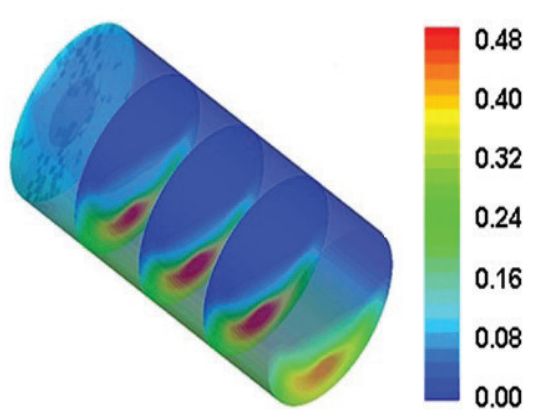

Gidaspow

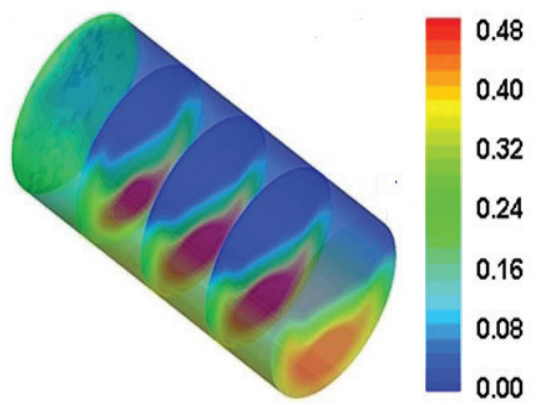

Gidaspow

(b) at $\mathrm{C} v f=20 \%$

Figure 2. Solid concentration contours at $V_{m}=2 \mathrm{~ms}^{-1}$ for (a) $C_{v f}=10 \%$ and $C_{v f}=20 \%$. 


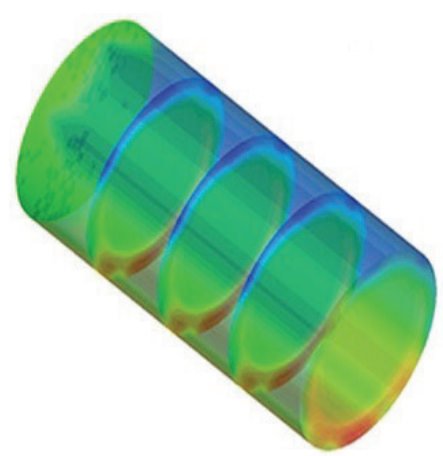

Syamlal - Obrien

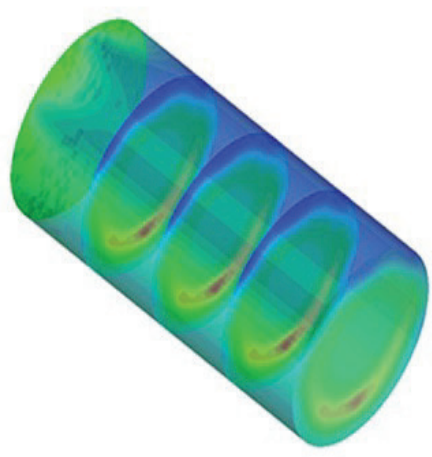

Syamlal - Obrien

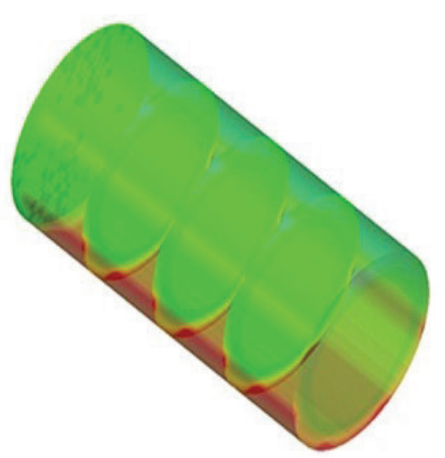

Schillar - naumann

(a) at $\mathrm{C} v f=10 \%$

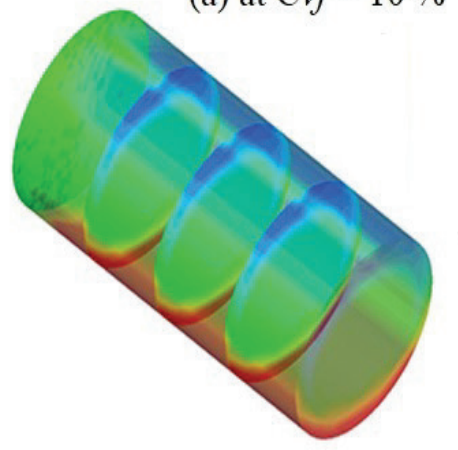

Schillar - naumann

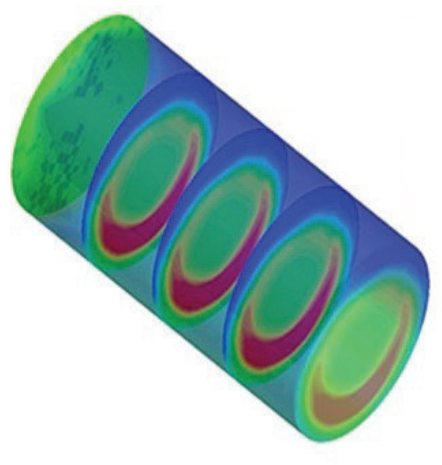

Gidaspow

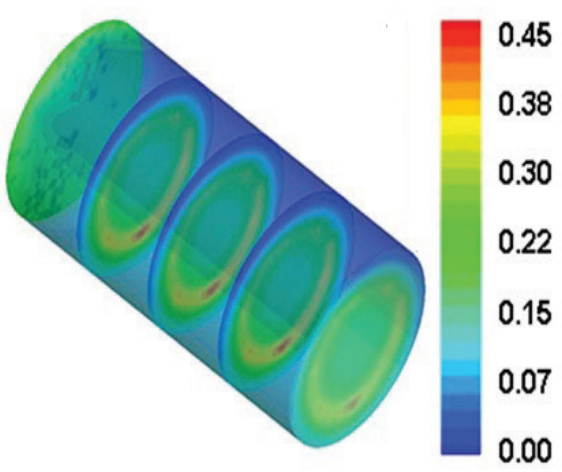

Gidaspow

(b) at $\mathrm{C} v f=20 \%$

Figure 3. Solid concentration contours at $V_{m}=5 \mathrm{~ms}^{-1}$ for (a) $C_{v f}=10 \%$ (b) $C_{v f}=20 \%$.

\subsection{Concentration Profiles}

Figure 4 depicts the solid concentration profiles in x-y plane at a distance, $\mathrm{x}=3.7 \mathrm{~m}$ from the pipe inlet at $C_{v f}=$ $10 \%$ and $=20 \%$ for chosen drag models. It is found that the solid concentration for all drag models diminishing from bottom to top of the pipeline. At low concentration, i.e., $C_{v f}=10 \%$ all drag models predict distinct concentration profile as depicted in figure 4(a). However, the drag models Gidaspow and Syamlal O'Brien nearly demonstrates the similar outcomes at $C_{v f}=20 \%$ as depicted in figure 4(b). Moreover, the drag models Gidaspow and Syamlal O'Brien nearly demonstrate similar outcomes at all velocity and solid concentration aside from at $V_{m}=5 \mathrm{~ms}^{-1}$ and $C_{v f}=10 \%$ (figure 7(a)) as depicted in figures 5, 6, and 7. Furthermore, the drag model Schiller-Naumann demonstrates the greater deviation from the other two models at all velocity and solid concentration range. 


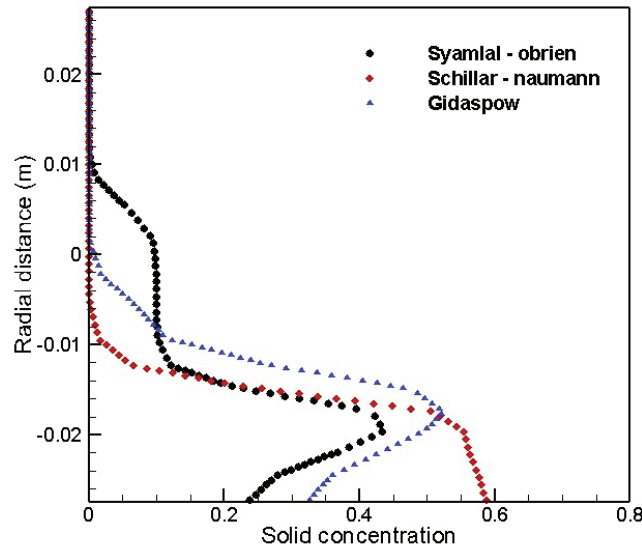

(a)

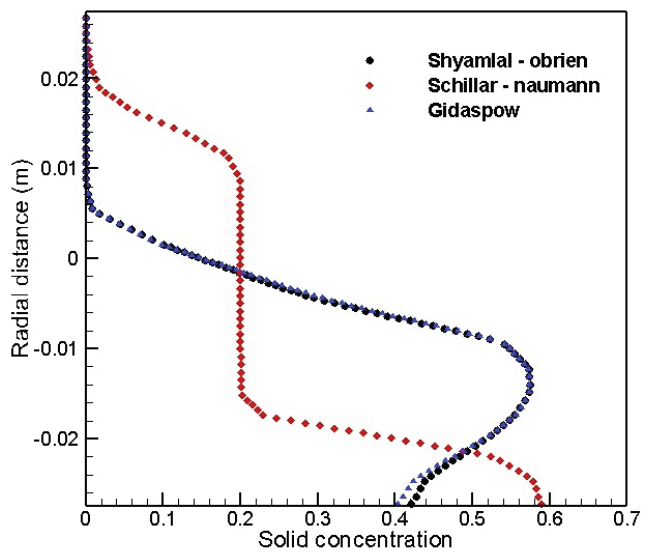

(b)

Figure 4. Solid concentration distribution at $V_{m}=2 \mathrm{~ms}^{-1}$ (a) $C_{v f}=10 \%$ and (b) $C_{v f}=20 \%$.

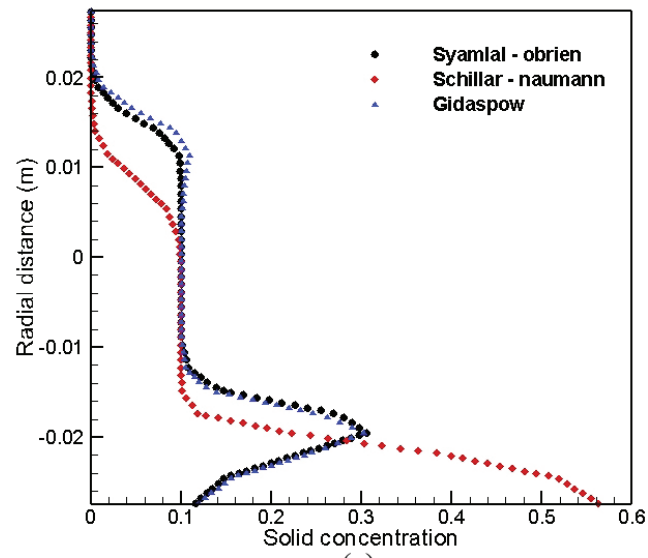

(a)

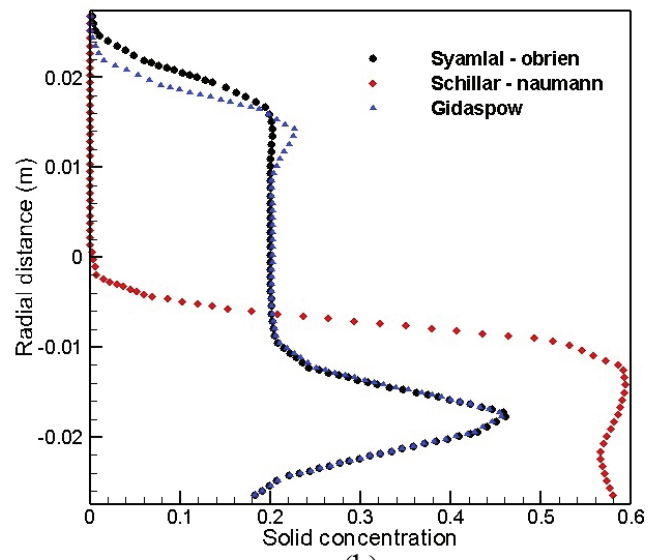

(b)

Figure 5. Solid concentration distribution at $V_{m}=3 \mathrm{~ms}^{-1}$ (a) $C_{v f}=10 \%$ and (b) $C_{v f}=20 \%$.

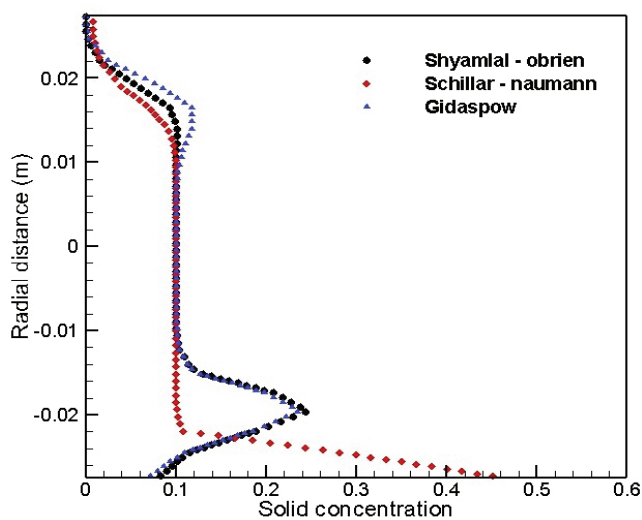

(a)

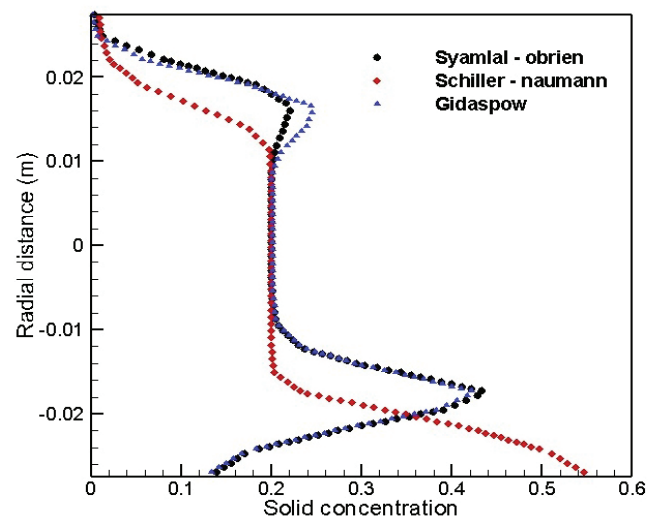

(b)

Figure 6. Solid concentration distribution at $V_{m}=4 \mathrm{~ms}^{-1}$ (a) $C_{v f}=10 \%$ and (b) $C_{v f}=20 \%$. 


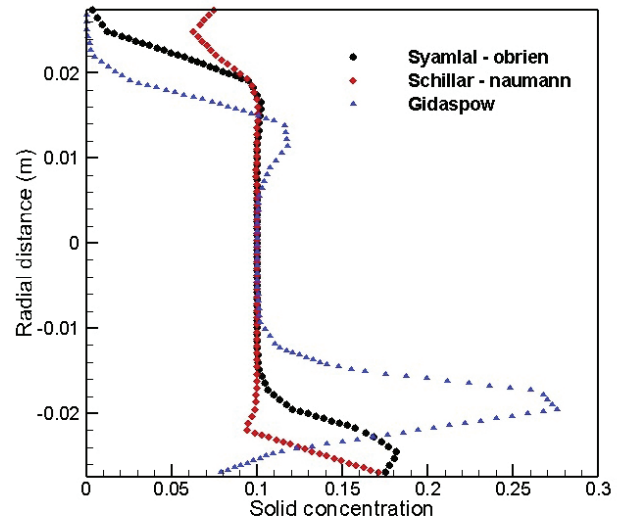

(a)

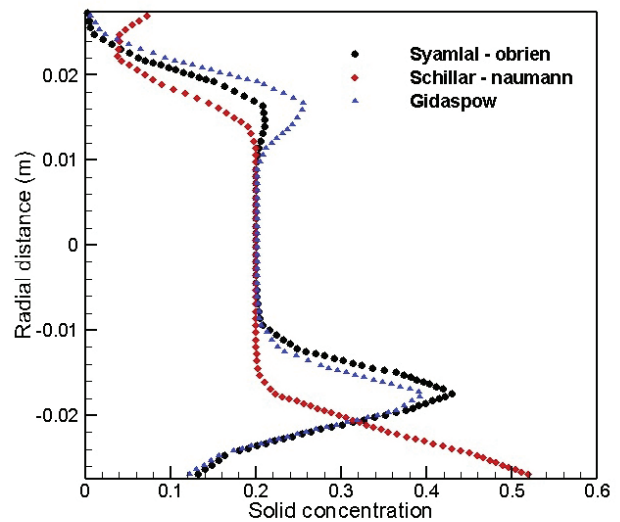

(b)

Figure 7. Solid concentration distribution at $V_{m}=5 \mathrm{~ms}^{-1}$ (a) $C_{v f}=10 \%$ and (b) $\mathrm{C}_{\mathrm{vf}}=20 \%$.

\section{MODEL VALIDATION}

The aftereffects of computed pressure drop for different drag models are validated with the published data by Kaushal et al. (2007) at solid concentration range, $C_{v f}=10 \%$ and $20 \%$ as depicted in figure 8 (a) and (b), respectively. The simulated outcomes show that the only Syamlal O'Brien drag model provides the accurate and meticulous results with the published data compared to other drag models. However, Gidaspow drag model outcomes the greater pressure drops at higher velocities across the slurry pipeline than Syamlal O'Brien and Schiller-Naumann drag models. Consequently, it is not appropriate for the transportation of slurry flow through pipeline at higher velocities. Additionally, Schiller-Naumann drag model outcomes the low pressure drops across the slurry pipeline, but it is not in synchronism with the experimental pressure drop line. In this way, Syamlal O'Brien drag model in conjunction with Eulerian two-phase turbulence model is recommended for the transportation of slurry flow through pipeline framework.

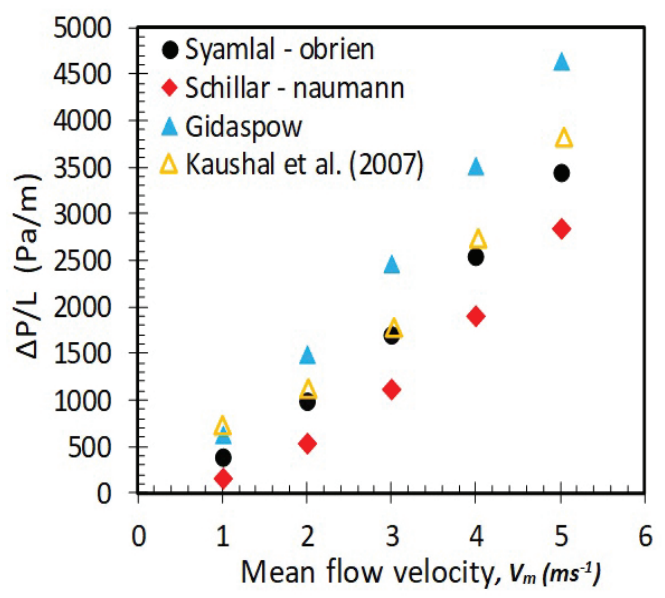

(a)

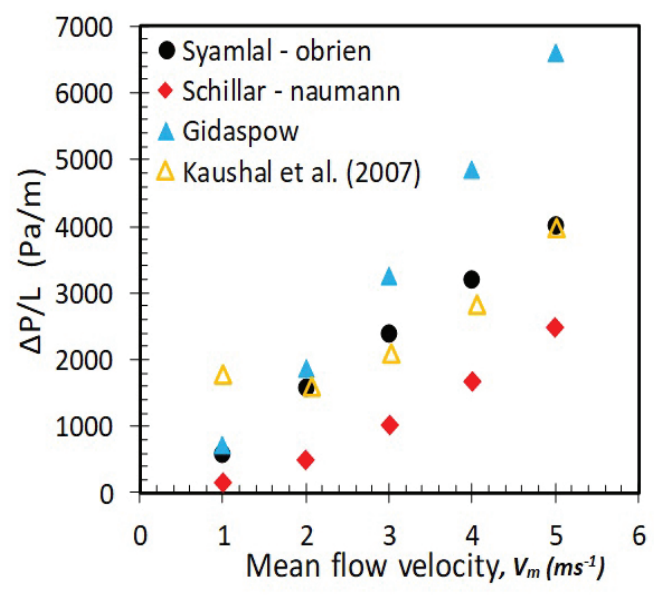

(b)

Figure 8. Validation of pressure drop with different drag model (a) $C_{v f}=10 \%$; (b) $C_{v f}=20 \%$. 


\section{CONCLUSION}

In light of the investigations on $0.0549 \mathrm{~m}$ diameter glass-beads slurry pipeline using commercial CFD software for computational drag models, the following conclusions have been drawn:

- It is observed that Eulerian two-phase model using RNG k- $\varepsilon$ turbulence closure gives the more appropriate and meticulous predictions of the solid concentration distribution and pressure drop for chosen drag models.

- The high solid concentration zone is situated closer to the pipe base for all drag models at all velocities and solid concentration range. However, the solid concentration zone is slightly away from the pipe base for Syamlal O'Brien and Gidaspow drag model while it touches the pipe base for Schiller-Naumann drag model.

- The consequences of solid concentration profile by Syamlal O'Brien and Gidaspow models are practically same in the lower half section of the pipe except at $V_{m}=5 \mathrm{~ms}^{-1}$ and $C_{v f}=10 \%$. Nonetheless, the aftereffects of solids concentration profile anticipated by Schiller-Naumann show more noteworthy from the other two models.

- The pressure drops increase with the increase in solid particulates concentration and stream velocity for all drag models. Nonetheless, the pressured drop by Gidaspow model is higher in magnitude as contrasted with the anticipated pressure drop by Syamlal O'Brien and Schiller-Naumann drag models.

- The obtained results of anticipated pressure drop by Syamlal O'Brien drag model are likened with the available published data and found in synchronism with that.

The comparison of the distinctive drag models utilizing Eulerian multiphase RNG k- $\varepsilon$ turbulence closure demonstrates the practical utility and high designing capability of slurry pipeline framework. In this manner, Eulerian multiphase RNG k- $\varepsilon$ turbulence model in conjunction with Syamlal O'Brien model is recommended for the transportation of slurry flow through pipeline framework. The work carried by the authors can further be extended on the bend pipe at higher concentration using the drag models investigated in this paper.

\section{REFERENCES}

O'Brien, M.P. 1933. Review of the theory of turbulent flow and its relations to sediment transportation. Transactions American Geophysical Union 14:487-491. DOI:10.1029/TR014i001p00487.

Rouse, H. 1937. Modern conceptions of the mechanics of fluid turbulence. Transactions ASCE 102:463-505.

Ismail, H.M . 1952. Turbulent transfer mechanism and suspended sediment in closed channels. Transactions ASCE 117:409-446.

Ling, J, Skudarnov, P.V., Lin, C.X. \& Ebadian, M.A. 2003. Numerical investigations of liquid solid slurry flows in a fully developed turbulent flow region. The International Journal of Heat and Fluid Flow 24:389398. DOI:10.1016/S0142-727X(03)00018-3

Kaushal, D.R. \& Tomita, Y. 2007. Experimental investigation for near-wall lift of coarser particles in slurry pipeline using $\gamma$-ray densitometer. Powder Technology 172(3):177-187. DOI:10.1016/j.powtec.2006.11.020

Lahiri, S.K. \& Ghanta, K.C. 2008. Prediction of pressure drop of slurry flow in pipeline by hybrid support vector regression and genetic algorithm model. The Chinese Journal of Chemical Engineering 16(6):841-848. DOI:10.1016/S1004-9541(09)60003-3

Monteiro, A.C. \& Bansal, P.K. 2010. Pressure drop characteristics and rheological modeling of ice slurry flow in pipes. International journal of refrigeration 33(8):1523-1532. DOI:10.1016/j.ijrefrig.2010.09.009

Majumder, A.K. 2015. Modeling fluid-particle interactions in flowing film type gravity concentrators. Journal of Engineering Research 4(3):1-16. 
Assefa, K.M. \& Kaushal, D.R. 2017. A new model for the viscosity of highly concentrated multi-sized particulate Bingham slurries. Particulate Science and Technology 35(1):77-85. DOI:10.1080/02726351.2015.1131789

Nayak, B.B. \& Chatterjee, D. 2018. Assessment of Mixture and Eulerian Multiphase Models in Predicting the Thermo-Fluidic Transport Characteristics for Fly Ash- Water Slurry Flow in Straight Horizontal Pipeline. Heat Transfer Engineering 1-14. DOI:10.1080/01457632.2018.1436670

Singh, J.P., Kumar, S. \& Mohapatra, S.K. 2018. Simulation and optimization of coal-water slurry suspension flow through $90^{\circ}$ pipe bend using CFD. International Journal of Coal Preparation and Utilization 1-23. DOI:10.1080/19392699.2018.1488693

Rabiei, M., Yi, Y., Bayat, A. \& Cheng, R. 2018. Simple methods for fluidic drag estimation during pipe installation via HDD. Tunnelling and Underground Space Technology 76: 172-176. DOI:10.1016/j.tust.2018.03.007

Xu, L., Huang, C.X., Huang, Z.F., Sun, Q. \& Li, J. 2018. Numerical simulation of flow and melting characteristics of seawater-ice crystals two-phase flow in inlet straight pipe of shell and tube heat exchanger of polar ship. Heat and Mass Transfer 54(11):3345-3360. DOI: 10.1007/s00231-018-2344-0

Li, M.Z., He, Y.P., Liu, Y.D. \& Huang, C. 2018. Effect of interaction of particles with different sizes on particle kinetics in multi-sized slurry transport by pipeline. Powder Technology 338:915-930. DOI:10.1016/j.powtec.2018.07.088

Cai, L., Liu, Z., Mi, S., Luo, C., Ma, K., Xu, A. \& Yang, S. 2019. Investigation on flow characteristics of ice slurry in horizontal $90^{\circ}$ elbow pipe by a CFD-PBM coupled model. Advanced Powder Technology 30(10):2299-2310. DOI:10.1016/j.apt.2019.07.010

Liu, L., Fang, Z., Qi, C., Zhang, B., Guo, L. \& Song, K.I. 2019. Numerical study on the pipe flow characteristics of the cemented paste backfill slurry considering hydration effects. Powder technology 343:454-464. DOI:10.1016/j.powtec.2018.11.070

Ting, X., Miedema, S.A. \& Xiuhan, C. 2019. Comparative analysis between CFD model and DHLLDV model in fully-suspended slurry flow. Ocean Engineering 181:29-42. DOI:10.1016/j.oceaneng.2019.03.065

Singh, K.P., Kumar, A. \& Kaushal, D.R. 2019. Experimental investigation on effects of solid concentration, chemical additives, and shear rate on the rheological properties of bottom ash (BA) slurry. International Journal of Coal Preparation and Utilization 1-14. DOI:10.1080/19392699.2019.1636787 